\title{
Caracterización molecular de poblaciones venezolanas de Sigmodon hirsutus (Rodentia: Cricetidae)
}

\author{
Janeth Lessmann ${ }^{1,2}$, Jazzmín Arrivillaga $^{1}$ \& Marisol Aguilera ${ }^{2 *}$ \\ 1. Universidad Simón Bolívar. Laboratorio de Genética de poblaciones y Ecología Molecular de Insectos, 1080-A \\ Caracas, Sartenejas, Baruta, Edo. Venezuela; lessmann@gmail.com, jarrivillaga@usb.ve. \\ 2. Universidad Simón Bolívar. Laboratorio de Biología Evolutiva, 1080-A Caracas, Sartenejas, Baruta, Edo. Miranda, \\ Venezuela; maguiler@usb.ve, janeth.lessmann@gmail.com \\ * Correspondencia.
}

Recibido 21-V-2010. Corregido 16-XII-2010. Aceptado 18-I-2011.

\begin{abstract}
Molecular characterization of Sigmodon hirsutus (Rodentia: Cricetidae) populations in Venezuela. Recent phylogenetic studies based on cytochrome b gene sequence, have determined that the species historically known as Sigmodon hispidus (Rodentia) from South America comprises a species S. hirsutus of paraphyletic origin. The aim of this study was to test the hypothesis that populations from Venezuela, represent the sensu strict form, ancestral haplotypes, and monophyletic subspecies. For this, 12 individual sequences from three localities of different biogeographic regions in Venezuela were evaluated and sequenced based on cyto b. Additionally, the sequences were used to develop a cladistic analysis and genetic distance calculations, and to compare this information with two individual sequences of Sigmodon specimens available in Genbank. Phylogenetic analyses show that the three populations of $S$. hirsutus of Venezuela form an ancestral and monophyletic subclade supported by high bootstrap values and significant genetic distance between subclade within the S. hirsutus. Besides, the existence of two lineages suggests two subspecies, S. hirsutus hirsutus from Venezuela, and S. hirsutus mexicanus from Mexico-Central America, but, both species need formal description. Rev. Biol. Trop. 59 (2): 795-807. Epub 2011 June 01.
\end{abstract}

Key words: Sigmodon hirsutus, Venezuela, cyto $b$, parsimony analysis, taxonomy.

Estudios filogenéticos indican que miembros del género Sigmodon (Familia: Cricetidae) comúnmente conocidos como "roedores algodoneros", constituyen el grupo más ancestral de los Sigmodontinos, subfamilia que incluye a todas las especies de roedores de Suramérica y algunas de Centro y Norteamérica (Smith \& Patton 1999, D’Elía 2003, Musser \& Carlenton 2005). Este género se distribuye desde las praderas y sabanas de Iowa y Nebraska, en Estados Unidos, hasta el norte de Suramérica, específicamente desde Colombia hasta Perú, y al este hasta Surinam (Voss 1992). Un aspecto taxonómico importante en Sigmodon es la poca variación de los caracteres morfológicos entre la mayoría de las especies de este género, por lo que ha sido difícil determinar entidades taxonómicas discretas y las relaciones entre éstas basado en la morfología (Voss 1992, Peppers et al. 2002).

Actualmente, se reconocen 14 especies de Sigmodon y una de ellas es Sigmodon hispidus, la cual ha presentado continuas discusiones taxonómicas (Musser \& Carlenton 2005). Fue descrita por Say \& Ord en 1825 en la localidad de Johns River, Florida, Estados Unidos, y basado en su homogeneidad morfológica se nominó como una especie simple distribuida en la mitad inferior de Estados Unidos (por debajo de $\operatorname{los} 40^{\circ}$ de latitud norte), Centroamérica y el norte de Colombia y Venezuela (Cameron $\&$ Spencer 1981). Para esta especie, al menos 
28 subespecies han sido nominadas, diez para Norteamérica, once para México y siete para Centro y Suramérica (Cabrera 1961, Carroll et al. 2005). Las subespecies que han sido referidas para Suramérica de $S$. hispidus son: S. h. peruanus, noreste de Perú, $S$. h. hispidus puna, Isla de Puna en la costa de Ecuador, $S . h$. simonsi, costa desértica del noroeste de Perú, $S$. h. chonensis, oeste de Ecuador entre los Andes y el Pacifico, $S$. h. inopinatus, Andes de Ecuador, $S . h$. bogotensis, valles interandinos del oeste de Colombia y $S$. h. hirsutus, noreste de Colombia y norte de Venezuela (Cabrera 1961).

En Venezuela, S. hispidus ha tenido su particular historia taxonómica, puesto que inicialmente, Burmeister en 1854 describe a Lasiomys hirsutus en Maracaibo, estado Zulia, mientras que Thomas en 1914, basado en una revisión morfológica del holotipo, concluye que Lasiomys hirsutus pertenece al género Sigmodon y la renombra como Sigmodon hirsutus. Finalmente, Cabrera (1961) establece de forma automática para Venezuela la ocurrencia de la subespecie $S$. hispidus hirsutus y sugiere la sinonimia de ésta con $S$. hirsutus.

Voss (1992) realiza una revisión basada en los caracteres morfológicos de casi 1300 ejemplares de Sigmodon de Suramérica, y concluye que existen cuatro especies diagnosticables. La primera de ellas es $S$. asltoni, sin previo conflicto taxonómico; las subespecies $S$. $h$. peruanus, $S$. h. chonensis, $S . h$. simonsi y $S . h$. puna conformarían la especie $S$. peruanus; y $S$. $h$. inopinatus es elevada a especie ( $S$. inopinatus). Los caracteres evaluados de S. h. bogotensis y $S$. h. hirsutus no presentaron suficientes diferencias con los ejemplares de S. hispidus de Florida (Estados Unidos), además de compartir cariotipos idénticos en número y morfología. Por lo tanto, estos roedores de Venezuela y Colombia continuaron refiriéndose como $S$. hispidus sin sugerirse el nivel de subespecie para esta área geográfica.

Linares en 1998 retoma la clasificación de Cabrera (1961) y reporta a los ejemplares de S. hispidus de Venezuela como la subespecie $S$. hispidus hirsutus, sin señalar si representan una variante fenotípica con respecto a otros $S$. hispidus del continente. La distribución de $S . h$. Hirsutus, según éste autor incluye la Cordillera Central, Lago de Maracaibo, ambas vertientes de Los Andes, Sistema Coriano y los Llanos entre 200 y $1580 \mathrm{~m}$ de altura.

Hay evidencias significativas sobre la divergencia genética dentro de la especie Sigmodon hispidus, aportadas por los resultados de electroforesis con suero sanguíneo, en donde se detectaron cinco patrones de transferrina entre ejemplares de Sigmodon hispidus de distintas regiones de Norte y Centroamérica (Dalby \& Lillevik 1969). Por otra parte, estudios de hibridización de ADN indicaron eventos de divergencia entre subespecies de S. hispidus del Norte y Suramérica, comparables con los correspondientes entre $S$. hispidus y $S$. arizonae y S. ochrognathus (Dickerman 1992).

Por consiguiente, se han propuesto cambios en la taxonomía de Sigmodon hispidus basados en la secuencia del gen de Citocromo $b$ del ADN mitocondrial (Peppers \& Bradley 2000, Peppers et al. 2002). En estos estudios se incluyeron nueve subespecies de S. hispi$d u s$, y como representantes de la distribución en Suramérica (Norte de Colombia y Norte de Venezuela) se analizaron dos ejemplares de S. hispidus hirsutus de la localidad de Sarare, Estado Lara, Venezuela. Los resultados por métodos de máxima verosimilitud y máxima parsimonia indicaron que el taxón históricamente referido como S. hispidus está compuesto por un ensamblaje parafilético de tres especies distintas, correspondientes a Estados Unidos, México y Centro-Suramérica. A raíz de estos resultados, las poblaciones de S. hispidus de Estados Unidos mantuvieron dicha nominación, por ley de prioridad, mientras que $S$. toltecus se asignó para México. Las poblaciones de Centro y Suramérica se nominaron como $S$. hirsutus, y la forma sensu estricto S. hirsutus hirsutus se encontraba restringida geográficamente a Suramérica.

Estudios posteriores han ampliado la distribución de S. hirsutus a México (Chiapas y Oxaca), Honduras y Nicaragua, ya que ejemplares colectados en estas áreas correspondieron genéticamente a esta especie (Bradley et 
al. 2008). A pesar de que los nuevos estudios se han enfocado en incluir más ejemplares del "grupo de especies hispidus" (S. hispidus, S. toltecus y $S$. hirsutus) de distintas localidades para evaluar los límites geográficos de las nuevas taxas propuestas, dichos trabajos no han analizado nuevas muestras de Suramérica (Carroll \& Bradley 2005, Carroll et al. 2005, Bradley et al. 2008).

Esta situación taxonómica sobre $S$. hirsutus hirsutus demanda ampliar el conocimiento de su distribución geográfica en Suramérica y de sus características morfológicas y genéticas. Es imperativo incluir en los análisis a otras poblaciones de Venezuela y Colombia con el fin de evaluar la hipótesis filogenética propuesta por Peppers et al. (2002). Adicionalmente, la resolución de la taxonomía y sistemática del grupo de especies hispidus tiene implicaciones epidemiológicas en Venezuela, puesto que diversos estudios han señalado a $S$. hispidus como reservorio de Trypanosoma cruzi y de Leishmania spp. (De Lima et al. 2002, De Lima et al. 2006).

En el presente trabajo se generó una matriz de datos con secuencias publicadas y disponibles en el Genbank para el género Sigmodon, junto con muestras adicionales obtenidas de ejemplares de S. hirsutus de Venezuela, con el propósito de responder las siguientes preguntas: ¿Cómo son las diferencias existentes en la composición nucleotídica de las secuencias estudiadas dentro y entre las poblaciones de Sigmodon hirsutus? ¿Cómo es la homología de las mismas con respecto a las secuencias de $S$. hirsutus hirsutus de Sarare (Estado Lara)? En caso de existir divergencia nucleotídica entre las secuencias de estas poblaciones ¿Éstas podrían explicarse mediante biogeografía? ¿Tales diferencias tendrían implicaciones a nivel taxonómico?

\section{MATERIALES Y MÉTODOS}

Se recolectaron 12 ejemplares identificados morfológicamente como S. hispidus, provenientes de dos localidades de Venezuela: Valle de Sarteneja $\left(10^{\circ} 24^{\prime} 50^{\prime \prime} \mathrm{N}-66^{\circ} 53^{\prime} 28^{\prime \prime} \mathrm{W}\right)$ Estado Miranda a $1286 \mathrm{msnm}$ (material colectado en 2008, que se utilizó como referencia morfológica y de cada ejemplar se tomó una muestra de músculo pectoral para extracción de $\mathrm{ADN}$, que se preservó en seco a $-20^{\circ} \mathrm{C}$ ) y Sierra de San Luis $\left(11^{\circ} 07^{\prime} 05^{\prime \prime} \mathrm{N} 69^{\circ} 41^{\prime} 01^{\prime \prime} \mathrm{W}\right)$, Estado Falcón a $702 \mathrm{msnm}$ (material colectado en 2006, que se utilizó como referencia morfológica y de cada ejemplar se tomó una muestra de músculo pectoral para extracción de ADN, que se preservó en etanol y se refrigeró).

El ADN del material preservado en seco fue extraído de forma individual empleando el método de Bender et al. (1983), mientras que para el material preservado en etanol se empleó el método Golczer \& Arrivillaga (2008), ambos protocolos están basados en la precipitación el ADN con sales, SDS y etanol. El ADN extraído se amplificó vía reacción en cadena de la polimerasa (PCR) mediante un termociclador PTC-100, MJ Research INC. Se emplearon dos juegos de cebadores para amplificar la región del gen que codifica para citocromo $b(810 \mathrm{bp})$ de Leite \& Patton (2002):

\section{MVZ005: (+) 5'CGAAGCTTGATATGAAAAACCATCGTTG‘3 MVZ016: (-) 5'AAATAGGAARTATCAYTCTGGTTTRAT'3}

Para las amplificaciones por PCR se utilizaron los siguientes volúmenes de reactivos: $5 \mu \mathrm{L}$ de agua libre de nucleasas, $5 \mu \mathrm{L}$ de FlexiBuffer 5X Promega ${ }^{\circledR}, 4 \mu \mathrm{L}$ de cloruro de magnesio $2.5 \mathrm{mM}$ Promega ${ }^{\circledR}, 0.5 \mu \mathrm{L}$ de nucleótidos $(100 \mathrm{mM}), 2.5 \mu \mathrm{L}$ de cada cebador $(1 \mathrm{pmol} / \mathrm{uL}$ de PCR), $0.5 \mu$ L de GoTaq Polimerasa Promega ${ }^{\circledR}$ y $5 \mu \mathrm{L}$ de muestra de extracción de ADN, para un volumen final de $25 \mu \mathrm{L}$. Los parámetros empleados para la amplificación en el termociclador fueron los siguientes: $94^{\circ} \mathrm{C} / 5 \mathrm{~min}$, 39 ciclos de $94^{\circ} \mathrm{C} / 30 \mathrm{~s}, 48^{\circ} \mathrm{C} / 30 \mathrm{~s}, 72^{\circ} \mathrm{C} / 45 \mathrm{~s}$ y $72^{\circ} \mathrm{C} / 45 \mathrm{~s}$. Posteriormente, en un gel de agarosa al $1.2 \%$ se visualizaron los productos 
amplificados y se usó para la verifición del peso del fragmento obtenido mediante el uso de un marcador de referencia de 100 pares de bases.

Los productos de PCR fueron purificados y secuenciados de forma automática por el Centro de Secuenciación y Análisis de Ácidos Nucleicos (CESSAN) en el Instituto Venezolano de Investigación Científica (IVIC), enviando $10-15 \mu \mathrm{L}$ de producto de PCR y $5 \mathrm{pmol} / \mu \mathrm{L}$ de los dos cebadores de cada juego.

Las secuencias obtenidas a partir de citocromo $b$ para las poblaciones de Venezuela fueron introducidas en el programa BLAST Basic Local Alignment Search Tool (Altschul et al. 1990), para evaluar que el fragmento amplificado correspondiera a la región de citocromo $b$ y que presentara máxima similitud con las secuencias de ejemplares del género Sigmodon, disponibles en el Genbank.

Para los análisis filogenéticos se incluyeron en la matriz total de datos, las secuencias amplificadas de las muestras de Venezuela y además se incluyeron 42 secuencias del gen de citocromo $b$ de ejemplares de distintas especies de Sigmodon reportados en los estudios de Peppers \& Bradley (2000), Peppers et al. (2002), Carroll \& Bradley (2005), Carroll et al. (2005) y Bradley et al. (2008), todas ellas disponibles en Genbank. El grupo externo escogido para este estudio fue Peromyscus leucopus (código de acceso Genbank AF131926), propuesto por Peppers et al. (2002).

Los ejemplares cuyas secuencias de ADN fueron consultadas para este estudio son listados a continuación con el número de museo o recolector, seguido del código de acceso al Genbank entre paréntesis. Las abreviaciones de los museos son las siguientes: University of Texas Medical Branch at Galveston ( $\mathrm{T}$ and FSH), Natural Sciences Research Laboratory, Museum of Texas Tech University (TTU y TK); The Museum of Southwestern Biology, University of New México (NK), Royal Ontario Museum (FN), Brigham Young University (BYU), Abilene Christian University Natural History Collection (ACUNHC), Royal Ontario Museum (ROM) y John C. Patton (JCP). S. alstoni: Portuguesa, Venezuela (T
2140, AF293396; T 2142, AF293397). S. alleni vulcanis: Michoacán, México (TK 45276, AF155425). S. arizonae major: Sonora, México (NK 6502, AF293398; TK 70928, AF155423). S. fulviventer dalquesti: Texas, Estados Unidos (TK 29915, AF293399). S. fulvivente fulviventer: Durango, México (TK 48915, AF293400). S. leucotis leucotis: Durango, México (TK 70798, AF293401). S. mascotensis mascotensis: Michoacán, México (JCP 1061, AF296188; JCP1020, AF155424). S. ochrognathus: Durango, México (TK 48609, AF155422; ACUNHC 500, AF155592). S. peruanus: El Oro, Ecuador (NK 37848, AF293395). S. toltecus furvus: Quintana Roo, México (FN 32695, AF293402). S. toltecus toltecus: Veracruz, México (NK 27055, AF155418). S. hispidus berlandieri: New Mexico, Estados Unidos (TK 47533, AF188198; TK 49611, AF155415). S. hispidus spadicipygus: Florida, Estados Unidos (FSH 33, AF1554 20). S. hispidus texianus: Oklahoma, Estados Unidos (TK 23428, AF155414). S. hirsutus hirsutus: Sarare, Venezuela (T 4632, AF155419). S. hirsutus borucae: Punta Arenas, Costa Rica (BYU 15259, AF108702). S. hirsutus chiriquensis: Chiriqui, Panamá (TK 22512, AF155416); Canal Zone Panamá (ROM 104228, AF425191). S. hirsutus spp.: Chiapas, México (TK 93324, AF425193, TTU 82799, AF425192; ROM 97578, AF425197; ROM 97584, AF425195; TTU 108157, AF425198; TTU 82798, AF425196; TK 138046, EU078398); Oaxaca, México (TK 93249, AF425194). S. hirsutus griseus: La Pax, El Salvador (TK 34810, AF155419); Olancho, Honduras (TK 102110, AY517529); Valle, Honduras (TK 101127, AY517530; TTU83794, EU073173); Francisco Morazán, Honduras (TTU 83741, AY517528); Olancho, Honduras (TTU84634, EU073172); Nueva Segovia, Nicaragua (TTU101442, EU073164; TTU100560, EU073168); Boaco, Nicaragua (TTU100570, EU073166; TTU100579, EU073165); Jinotega, Nicaragua (TTU108154, EU073167).

Para el alineamiento de las secuencias mencionadas se empleó el programa CLUSTALW, Multiple Sequence Alignment (Thompson et al. 1994) en donde se utilizó la inserción de 
gaps, con una penalidad por inserción de gaps de 10 y de 6.66 de penalidad por la creación de gaps de extensión, con el fin de garantizar un alineamiento más homólogo (Golczer \& Arrivillaga 2010).

Las secuencias alineadas fueron analizadas en el programa MEGA-Molecular Evolutionary Genetics Analisys version 4 (Tamura et al. 2007) para determinar la composición de bases, caracteres conservados, variables e informativos para parsimonia y las tasas de transversiones y transiciones.

De igual forma, las secuencias previamente alineadas se emplearon para construir un árbol de parsimonia se siguió la metodología de Peppers et al. (2002) con la finalidad de identificar genéticamente a los ejemplares de las poblaciones de Venezuela según la hipótesis filogenética propuesta por estos autores. Para el análisis de parsimonia realizado en el programa PAUP Versión 4 (Phylogenetic Analysis Using Parsimony) de Swofford (2001), no se consideraron los gaps como caracteres, se realizó una búsqueda heurística, con remuestreo tipo TBR (disección reconexión de ramas, para búsqueda del árbol más corto dentro de los más parsimoniosos), 100 replicaciones aditivas azarosas y 1000 réplicas de bootstrap (Carpenter 1996). Se efectuaron análisis con pesaje y sin pesaje sobre el índice de consistencia (CI) y se estimó el consenso estricto de los árboles más parsimoniosos obtenidos. Solo se realizó análisis cladístico, por ser un método deductivo, ampliamente usado en el grupo por diferentes autores lo que permite una comparación óptima y directa de los resultados. Además, los resultados y la inferencia biológica derivada de MP son similares a los obtenidos utilizando métodos probabilísticos, ML o análisis bayesiano (Peppers \& Bradley 2000, Caroll \& Bradley 2005, Bradley et al. 2008).

Las secuencias alineadas fueron analizadas mediante el programa MEGA para estimar las distancias genéticas y se empleó el modelo de sustitución nucleotídica TamuraNei (TN93), en el cual se distinguen dos tipos de transiciones que se dan en tasas diferentes, se asume que las transversiones se dan a la misma tasa, pero a una tasa diferente respecto a las transiciones (Tamura \& Nei 1993). Con las distancias genéticas obtenidas se estimó el tiempo de divergencia, tomando en cuenta que la tasa de evolución molecular para citocromo $b$ en roedores corresponde a un millón de años de divergencia por cada $3.5 \%$ de distancia entre las secuencias, según análisis propuesto por Peppers et al. (2002).

Mediante el Test de Tajima (Tajima 1993) disponible en el programa MEGA, se determinó el número de sitios diferentes y el número de sitios divergentes considerando las transiciones y transversiones por igual, entre grupos de secuencias de las poblaciones de Venezuela y otros linajes del grupo hispidus.

\section{RESULTADOS}

Ahora bien, se amplificó un fragmento de 810bp para un total de 12 muestras de campo. La búsqueda de similitud máxima entre las secuencias obtenidas y las disponibles en el Genbank, con el empleo del programa BLAST, evidenció la máxima similitud con las secuencias de S. hirsutus hirsutus de Sarare, Venezuela (código de acceso AF155419).

Por lo tanto se encontró que para cada población estudiada de Venezuela existe un solo haplotipo por localidad: Valle de Sartenejas (Haplotipo 1), Sierra de San Luis (Haplotipo 2) y Sarare (Haplotipo 3). Estos tres haplotipos, se diferencian entre sí por una máximo de siete posiciones nucleotídicas: P66 (C en Sartenejas Haplotipo vs T), P298 (G en Sarteneja Haplotipo vs A), P445 en Sarare Haplotipo vs C), P628 (C en Sarare Haplotipo vs T), P682 (T en Sartenejas Haplotipo vs C), P802 (C San Luis Haplotipo vs T), P807 (A en Sarare Haplotipo vs C).

En relación con la composición nucleotídica, se trabajó con un número total de 55 secuencias, con un promedio de $1073.3 \mathrm{bp}$ con 390 caracteres informativos para parsimonia, que es una proporción semejante a la obtenida por Peppers et al. (2002). No se encontraron diferencias significativas entre la composición de bases de las secuencias de los ejemplares de 
Venezuela con respecto a las reportadas para $S$. hirsutus hirsutus y otros ejemplares de $S$. hirsutus de Centroamérica y México.

El análisis filogenético realizado por el método de parsimonia con pesaje sobre el índice de consistencia y Peromyscus leucopus como único grupo externo produjo un solo árbol como el más parsimonioso (715.6 pasos, índice de consistencia 0.57 , índice de retención 0.77). En general, se observa el origen parafilético del grupo de especies hispidus (S. hirsutus, S.toltecus y S. hispidus), (Fig. 1). Con respecto

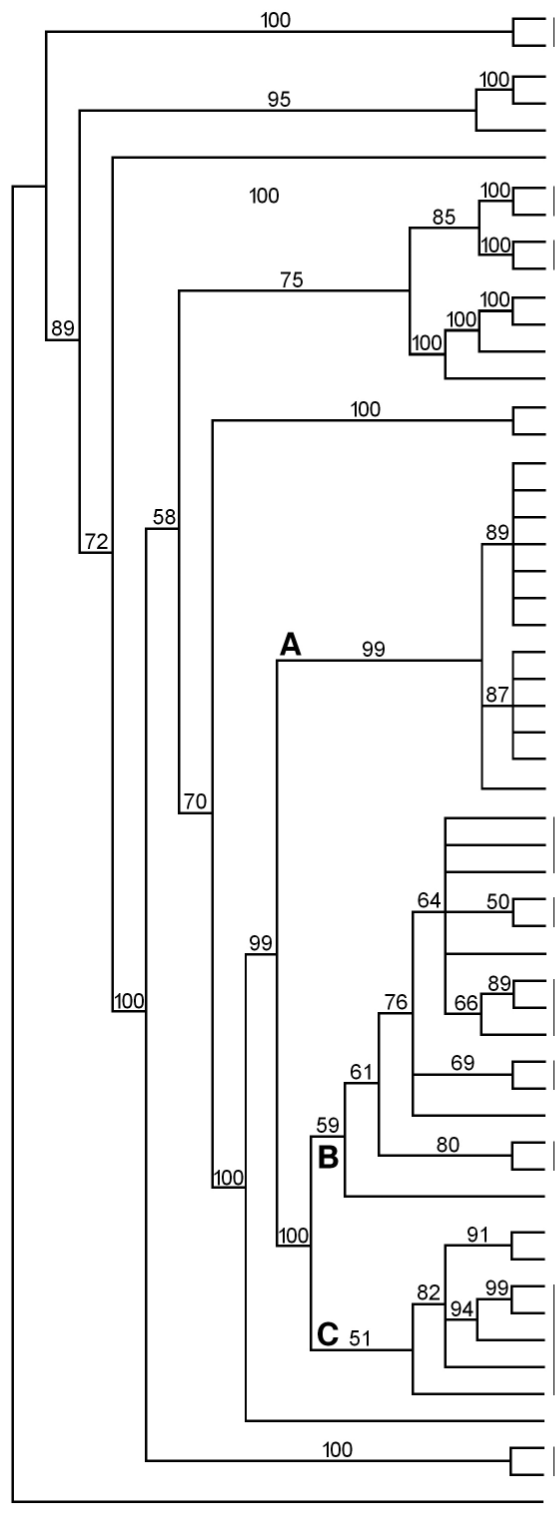

S. alstoni

S. fulviventer dalquesti

S. fulviventer fulviventer

$S$. leucotis leucotis

S. peruanus

S. arizonae major

S. mascotensis mascotensis

S. hispidus berlandieri

$S$. hispidus texianus

S. hispidus berlandieri

$S$. hispidus spadicipygus

S. toltecus furvus

$S$. toltecus toltecus

2213 Valle de Sartenejas

2214 Valle de Sartenejas

2215 Valle de Sartenejas

2217 Valle de Sartenejas

2218 Valle de Sartenejas

2220 Valle de Sartenejas

2221 Valle de Sartenejas

25342 Sierra de San Luis $~ N$

25343 Sierra de San Luis

25344 Sierra de San Luis

25378 Sierra de San Luis

25379 Sierra de San Luis $]$ I

S. hirsutus hirsutus Venezuela, Sarare

S. hirsutus griseus Nicaragua

S. hirsutus griseus Honduras

S. hirsutus griseus EI Salvador

S. hirsutus griseus Honduras

S. hirsutus griseus Nicaragua

S. hirsutus griseus Costa Rica

S. hirsutus chiriquensis Panamá

S. hirsutus spp. México, Chiapas

S. hirsutus spp. México, Chiapas

$S$. hirsutus spp. México, Oaxaca

S. hirsutus spp. México, Chiapas

S. alleni vulcanis

S. ochrognathus

Peromyscus leucopus

Fig. 1. Árbol filogenético de máxima parsimonia generado para especies del género Sigmodon, a partir del análisis pesaje sobre el CI, Permyscus leucopus como grupo externo. Número de árboles encontrados: 1; CI: 0.61; RI: 0.76; pasos: 784.

Fig. 1. Maximum parsimony phylogenetic tree generated for species of Sigmodon, from weighing on the CI analysis, Permyscus leucopus as outgroup. Number of trees found: 1, CI: 0.61, RI: 0.76, steps: 784. 
al clado de $S$. hirsutus, los veinticinco haplotipos de esta especie forman un grupo monofilético (Bootstrap $=99 \%$ ) relacionado con la especie hermana S. alleni. Dentro de este clado, tres subclados fueron identificados (A, B y C). El subclado A (Bootstrap=99\%) están contenidos los Haplotipos 1 y 2 de las poblaciones de Venezuela, los cuales forman una politomía con el Haplotipo 3 de Sarare, correspondiente a $S$. hirsutus hirsutus. El subclado B (Bootstrap $=59 \%$ ) contiene los ejemplares de Centroamérica y uno perteneciente a Chiapas, México, mientras que el subclado C (Bootstrap=51\%) presenta ejemplares de Chiapas y Oaxaca de México. Este último resultado no apoya la monofilia de los subclados B y C y evidencia un origen parafilético de las muestras del sur de México identificadas como S. hirsutus en su forma sensu lato, por lo que este grupo de haplotipos representan un único linaje.

Para S. hirsutus de Venezuela, las distancias genéticas intrapoblaciones obtenidas son iguales a cero, de manera que los ejemplares analizados dentro de cada localidad son idénticos con base en la composición nucleotídica, mientras que los valores interpoblacionales entre estos tres haplotipos estuvieron entre 0.5$0.6 \%$ (Cuadro 1). Las distancia intrasubclados para $S$. hirsutus indican que las poblaciones estudiadas de Venezuela tienen una homogeneidad genética mayor (subclado $\mathrm{A}=0.6 \%$ ), en comparación con aquellas de Centroamérica (subclado $\mathrm{B}=0.8 \%$ ) ó México (subclado $\mathrm{C}=5.3 \%$ ). Las distancias genéticas intersubclados estuvieron entre 3.7 y $6.3 \%$, las cuales sugieren tiempos de divergencia según el reloj molecular entre 1.06-1.80 millones de años. La distancia genética intraespecífica del grupo hispidus fue de $4.0 \%$ para S. hirsutus, de $2.9 \%$ para S. hispidus y de $3.0 \%$ para S. toltecus.

Los resultado del test de Tajima indican la ausencia de sitios divergentes entre las secuencias de las localidades de S. hirsutus hirsutus de Venezuela, mientras que al comparar secuencias de los tres subclados de S. hirsutus se obtuvo siempre un sitio de divergencia (Cuadro 2).

\section{DISCUSIÓN}

Las poblaciones del Valle de Sartenejas y de la Sierra de San Luis presentaron cada una un solo haplotipo para las $810 \mathrm{pb}$ del gen de Citocromo $b$, marcador molecular que comúnmente muestra variabilidad a nivel intrapoblacional en mamíferos (Kocher et al. 1989). Sin embargo, esta ausencia de variabilidad también ha sido señalada por otros autores para los roedores: Akodon cursos (Geise et al. 2001), Stenocephalemys sp., Praomys albipes (Lavrenchenko \& Verheyen 2006), y para $S$. alstoni, S. hirsutus hirsutus y $S$. mascotensis (Peppers et al. 2002). En el análisis de parsimonia, estos dos haplotipos (del Valle de Sartenejas y de la Sierra de San Luis) formaron un grupo monofilético junto con la secuencia de S. hirsutus hirsutus de Sarare, Estado Lara, con un alto valor de Bootstrap (99\%). Estos resultados indican que los ejemplares recolectados e identificados como S. hispidus en esas localidades corresponden a $S$. hirsutus hirsutus y constituyen el registro de dos nuevas localidades para la distribución de esta especie de acuerdo a éste gen.

En general, los tres haplotipos para $S$. hirsutus hirsutus de Venezuela pertenecientes a tres poblaciones aisladas geográficamente y a tres áreas biogeográficas: Cordillera de la Costa (Valle de Sartenejas), Sistema Coriano (Sierra de San Luis) y Cordillera Andina (Sarare), no evidencian divergencia nucleotídica por lo que no hay estructura filogenética dada a una politomia dura interna, lo cual indica la existencia de una historia evolutiva común. Esta homogeneidad o ausencia de estructura puede ser explicada por la presencia de poblaciones continuas (Linares 1998), no examinadas en este trabajo, que podrían mantener el flujo génico entre las poblaciones más periféricas. Además, para el caso de la especie S. hispidus, se ha registrado una movilidad importante de 13 metros por día en promedio avanzando frente a barreras naturales, características éstas que pueden aumentar la dispersión geográfica de la 
Distancia genética (Tamura-Nei) y tiempo de divergencia para las especies y grupos de Sigmodon

TABLE 1

Genetic distance (Tamura-Nei) and divergence time for species and groups of Sigmodon

\section{Grupo}

Distancia genética (\%)

Tiempo de divergencia (m.a.)

Distancias intrapoblacionales de S. hirsutus de Venezuela

S. hirsutus Valle de Sartenejas

S. hirsutus Sierra de San Luis

0

0

Distancias interpoblacionales de $S$. hirsutus de Venezuela

S. hirsutus Sierra de San Luis vs. S. hirsutus Valle de Sartenejas

0.6

0.17

S. hirsutus Sierra de San Luis vs. S. hirsutus Sarare

0.5

0.6

0.14

S. hirsutus Sarare vs. S. hirsutus Valle de Sartenejas

Distancias dentro de subclados de $S$. hirsutus

Dentro de $S$. hirsutus Venezuela (A)

Dentro de $S$. hirsutus Centroamérica (B)

Dentro de S. hirsutus México (C)

0.6

0.8

5.3

Distancias entre los subclados de $S$. hirsutus

S. hirsutus Venezuela (A) vs. S. hirsutus México (C)

6.3

3.7

4.2

4.5

S. hirsutus Venezuela (A) vs. S. hirsutus Centroamérica y México (B y C)

Distancias intraespecíficas

Dentro de $S$. hirsutus

Dentro de S. hispidus

4.0

2.9

3.0

Distancias interespecíficas

S. hirsutus Centroamérica y México (B y C) vs. S. alleni

8.8

7.0

13.0

$\begin{array}{lrr}\text { S. hirsutus vs. S. toltecus } & 12.6 & 3.63\end{array}$

$\begin{array}{lrr}\text { S. hispidus vs. S. toltecus } & 12.6 & 3.60\end{array}$

$\begin{array}{lrr}\text { S. hirsutus vs. S. alleni } & 8.1 & 2.14\end{array}$

21.7

S. hirsutus vs Peromyscus leucopus

$\begin{array}{cc} & - \\ & - \\ 0 & 0.17 \\ 0.6 & 0.14 \\ & 0.17\end{array}$

6

.3

1.80

1.06

1.17

0

3.9

2.51

S. hirsutus Venezuela (A) vs. S. alleni

S. hirsutus vs. S. hispidus

Se asume que cada $3.5 \%$ de diferencia corresponde a una divergencia de un millón de años. It is assumed that every $3.5 \%$ difference corresponds to a difference of one million years.

especie así como el flujo entre las poblaciones (Cameron \& Spencer 1981, Bowne et al. 1999).

Aunque, los resultados no muestran una estructura filogenética para las poblaciones de S. hirsutus hirsutus de Venezuela, parece existir una agrupación interna que muestra relación entre las distancias genéticas y las distancias geográficas. La población del Valle de Sartenejas ubicada a $316 \mathrm{~km}$ de la Sierra de San Luis y a $257 \mathrm{~km}$ de Sarare presenta una distancia genética de $0.6 \%$ con respecto a ambas poblaciones, mientras que Sarare y Sierra de San Luis, separadas por $156 \mathrm{~km}$, presentan una leve pero menor distancia genética de $0.5 \%$.

Con respecto a la composición nucleotídica de las poblaciones del Valle de Sartenejas 
CUADRO 2

Divergencia nucleotídica (Test de Tajima) entre secuencias-haplotipos

TABLE 2

Nucleotide divergence (Test Tajima) between sequences-haplotypes

\begin{tabular}{|c|c|c|c|}
\hline Grupo de secuencias & $\begin{array}{c}\mathrm{N}^{\circ} \text { de sitios idénticos } \\
\text { en las secuencias }\end{array}$ & $\begin{array}{c}\mathrm{N}^{\circ} \text { de sitios divergentes } \\
\text { entre las secuencias }\end{array}$ & $\begin{array}{l}\mathrm{N}^{\circ} \text { de sitios diferentes } \\
\text { únicos en la secuencia }\end{array}$ \\
\hline Valle de Sartenejas (Subclado A) & & & 3 \\
\hline Sierra de San Luis (Subclado A) & 803 & 0 & 2 \\
\hline Sarare (Subclado A) & & & 2 \\
\hline Valle de Sartenejas (Subclado A) & & & 1 \\
\hline Sarare (Subclado A) & 780 & 1 & 3 \\
\hline S. hirsutus Honduras (Subclado B) & & & 25 \\
\hline Valle de Sartenejas (Subclado A) & & & 2 \\
\hline Sarare (Subclado A) & 764 & 1 & 2 \\
\hline S. hirsutus México (Subclado C) & & & 41 \\
\hline S. hirsutus griseus Nicaragua (Subclado B) & & & 2 \\
\hline S. hirsutus México (Subclado C) & 1105 & 1 & 31 \\
\hline S. hirsutus griseus El Salvador (Subclado B) & & & 4 \\
\hline Valle de Sartenejas (Subclado A) & & & 14 \\
\hline S. hirsutus Honduras (Subclado B) & 707 & 6 & 6 \\
\hline S. hispidus texianus & & & 77 \\
\hline
\end{tabular}

y Sierra de San Luis, estas son similares a las reportada para S. hirsutus hirsutus de Sarare y para S. hirsutus de Centroamérica y México, las mismas apoyan la identificación genética de los ejemplares analizados como $S$. hirsutus. A su vez, la composición de las secuencias de la matriz total también se mantiene en proporciones similares a las señaladas por Peppers \& Bradley (2000) [T: 31.1\%, C: $28.9 \%$, A: $27.2 \%$ y G:12.8\%], Carroll \& Bradley (2005) [T: $31.2 \%, \mathrm{C}: 28.8 \%, \mathrm{~A}: 28.8 \%$ y G:11.2\%] que presentan el típico porcentaje bajo de guaninas de la región de citocromo $b$ en roedores (Irwin et al. 1991).

En el árbol filogenético resultante, el clado $S$. hirsutus está constituido por tres subclados. El primero de ellos contiene secuencias de ejemplares de Venezuela, subclado A; el segundo de Centroamérica mayoritariamente subclado B y el tercero de México, Oaxaca y Chiapas, subclado C. Sin embargo, solo se evidencia sitios de divergencia nucleotídica (Test de Tajima) entre las secuencias-individuos del subclado A con respecto al subclado B/C. El análisis de parsimonia evidencia sólo monofilia reciproca para el subclado A (Venezuela) basado en valores de bootstrap altos (99\%), mientras los subclados B y C están evidenciados por valores de bootstrap bajos (51 y $59 \%$ respectivamente). Adicionalmente, el origen parafilético de las secuencias de S. hirsutus spp. pertenecientes a México, Chiapas, no apoyan la hipótesis de un origen independiente para las poblaciones que integran los subclados B y C (Costa Rica, Honduras, Nicaragua y México) explicado probablemente por introgresión, lo que sugiere la existencia taxonómica de una misma entidad filogenética en México y Centroamérica.

El valor de la distancia genética entre $S$. hirsutus de Venezuela (subclado A) con respecto a $S$. hirsutus de Centroamérica y México (subclados B y C) fue de $4.5 \%$, que mucho más bajo que las distancias entre especies calculadas para este estudio (entre $8.1 \%$ y $13.0 \%$ ). No obstante, otros trabajos que emplearon 
citocromo $b$ han reportado distancias similarmente bajas entre especies de roedores del mismo género. Por ejemplo, para Peromyscus la distancia interespecífica varió entre $4 \%$ y $9.5 \%$ (Sullivan et al. 1997) y para las especies del género Oxymycterus los valores oscilaron desde $1.9 \%$ hasta $9.6 \%$ (Hoffmann et al. 2002). Igualmente, Bradley et al. (2008), señalan que este valor de distancia entre Venezuela y Centroamérica-México, si bien es bajo, indica algún nivel de aislamiento y probablemente estos dos grupos representen diferentes trayectorias evolutivas.

Según Bradley et al. (2008), la divergencia de Venezuela con respecto a Centroamérica y México puede deberse a la influencia de barreras existentes en el Istmo de Panamá. Sin embargo, Carroll et al. (2005) opinan que el Istmo de Panamá no es efectivo para separar a estas poblaciones, ya que en un estudio previo se determinó que ejemplares de $S$. hispidus del este y oeste de Estados Unidos (un territorio continuo) poseen una divergencia genética del $4.8 \%$, un poco mayor a la encontrada entre los subclados de Centro y Suramérica. Particularmente, nuestros resultados apoyan la hipótesis de Bradley et al. (2008).

La distancia dentro del subclado A, que agrupa haplotipos de la subespecie $S$. hirsutus de Venezuela $(0.6 \%)$ fue similar a la obtenida dentro del clado de Centroamérica, subclado B $(0.8 \%)$. En contraste, para el clado de México, Subclado C, sin contener subespecies, la distancia genética fue mucha más elevada (5.3\%), lo que sugiere que los ejemplares de $S$. hirsutus de Oaxaca y Chiapas de México, posiblemente se encuentran aislados por el establecimiento del Istmo Tehuantepec (Bradley et al. 2008). Para las poblaciones de Centroamérica, la alta homogeneidad nos sugiere que la identificación taxonómica de tres subespecies no es la más adecuada, según los resultados filogenéticos. Por otro lado, se obtuvo un alto valor de distancia entre los subclados de Centroamérica (B) y México (C) de $4.2 \%$, y entre estos clados y el de Venezuela de $6.3 \%$.

La distancia intraespecífica de S. hirsutus, de $4.0 \%$ es ligeramente mayor comparada con otras especies del género como $S$. hispidus (2.9\%) y S. toltecus (3.0\%). A su vez, otros estudios con base en Citocromo $b$ han estimado distancias intraespecíficas menores o semejantes a los reportados aquí. Por ejemplo, las distancia dentro de las especies de los Oryzomyinos fue de $3 \%$ en promedio (Myers et al. 1995), para cuatro especies del género Akodon fue de $0.5 \%$ a $3.95 \%$ (Patton \& Smith, 1992), para especies del grupo Peromyscus de $0.5 \%$ hasta $3.5 \%$ (Sullivan et al. 1997) y para el género Oxymycterus las variaciones fueron de $0 \%$ hasta $1.3 \%$ (Hoffmann et al. 2002). Por lo tanto, la distancia genética obtenida para S. hirsutus parece ser alta en estos términos, opinión apoyada por Carroll et al. (2005).

Consecuentemente, los valores relativamente altos para la distancia intraespecífica de $S$. hirsutus y para las distancias entre las poblaciones estudiadas de Venezuela, Centroamérica y México parecen apoyar la hipótesis de aislamiento entre estas tres poblaciones-subclados. Sin embargo, para evaluarla satisfactoriamente es necesario incluir más información genética de ejemplares de Suramérica, especialmente de poblaciones de Colombia, no estudiadas aún.

Al considerar el resultado del análisis de parsimonia los ancestros más inmediatos de $S$. hirsutus parecen ubicarse en Norteamérica. El tiempo de divergencia de $S$. hirsutus y de $S$. alleni (México) es de aproximadamente 2.14 millones de años, por lo que $S$. hirsutus representa una invasión relativamente reciente a Suramérica, que se puedo haber dado a través del Istmo de Panamá. Diferenciándose y aislándose posteriormente las poblaciones de Sur y Centroámerica de S. hirsutus durante el periodo cuaternario (1-1.80 millones de años).

Nuestros resultados concuerdan con la hipótesis de Pepper et al. (2002) quienes señalan que las poblaciones de $S$. hirsutus de Suramérica (con base en las muestras de Venezuela) son las más ancestrales, así que por ley de prioridad, los ejemplares de esta distribución están correctamente nominadas como la forma sensu stricto, S. hirsutus hirsutus. Esta afirmación es importante desde el punto de vista taxonómico, puesto que permiten generar una descripción 
formal de S. hirsutus con ejemplares de Venezuela (trabajo en progreso) por su distribución actual endémica.

Finalmente, los resultados de diferenciación genética y filogenética para la especie $S$. hirsutus hirsutus respecto al clado $\mathrm{B} / \mathrm{C}$, sugiere al menos la existencia de dos subespecies dentro de S. hirsutus: subespecie a) la forma sensu estricto de Venezuela y subespecie b) la forma Centro América-México. La subespecie b agrupa varias regiones geográfica, cuyos linajes han sido señalados por otros autores como al menos tres subespecies, sin embargo, los análisis muestran valores bajos de Bootstrap para los dos subclados internos B y C, y un origen parafilético para los haplotipos pertenecientes a las poblaciones de México, lo que invalida la existencia de varias subespecies, y su reducción taxónomica a una subespecie filogenética denominada en el presente trabajo como subespecie $S$. hirsutus mexicanensis.

\section{AGRADECIMIENTOS}

Los autores extienden su agradecimiento a Victor Romero, Tatiana Caldera, Raúl Vegas por su colaboración en el trabajo de campo. Victor Romero, Angela Martino, Juan Carlos Navarro, Emilio Herrera, Jorge Pérez, Roberto Cipriani por los comentarios al trabajo de tesis de J. Lessmann.

\section{RESUMEN}

Recientes estudios filogenéticos basados en la secuencia del gen del citocromo b, han determinado que la especie conocida históricamente como Sigmodon hispidus (Rodentia) en Suramérica incluye una especie $S$. hirsutus de origen parafilético. El objetivo de este estudio fue probar la hipótesis de que las poblaciones de Venezuela, representan la forma sensu estricto, los haplotipos ancestrales y una subespecie monofilética. La metodología consistió en un análisis cladístico y cálculos de distancia genética, a partir de secuencias de citocromo b en 12 individuos de tres localidades en Venezuela que pertenecen a diferentes regiones biogeográficas, y a su vez compararlas con las dos secuencias disponibles en Genbank de especies del género Sigmodon. Los análisis filogenéticos indican que las tres poblaciones de $S$. hirustus de Venezuela forman un subclado ancestral y monofilético con el apoyo de valores de bootstrap altos y con significativa distancia genética, entre subclados dentro de $S$. hirsutus. La existencia de dos subclados dentro de $S$. hirsutus sugiere dos subespecies, $S$. hirsutus hirsutus en Venezuela y S. hirsutus mexicanus en México y Centroamérica. Sin embargo, ambas subespecies necesitan una descripción formal.

Palabras clave: Sigmodon hirsutus, Venezuela, citocromo $b$, parsimonia análisis, taxonomía.

\section{REFERENCIAS}

Altschul, G.W., W. Miller, E.W. Myers \& D.J. Lipman. 1990. Basic local alignment search tool. J. Mol. Biol. 215: 403-10.

Bender, W., P. Spierer \& D.S. Hogness. 1983. Chromosomal walking and jumping to

isolate DNA from the Ace and rosy loci and the bithoraxcomplex in Drosophila melanogaster. J. Mol. Biol. 168: $17-33$.

Bowne, D.R., J.D. Peles \& G.W. Barrett. 1999. Effects of landscape spatial structure on movement patterns of the hispid cotton rat (Sigmodon hispidus). Landsc. Ecol. 14: 53-65.

Bradley, R.D., D.D. Henson \& N.D. Durish. 2008. ReEvaluation of the geographic distribution and phylogeography of the Sigmodon hispidus complex based on mitochondrial DNA sequence. Southwest Nat. 53: 301-310.

Burmeister, H. 1854. Ueber eine neue Ratte, Lasiomys hirsuta, von Maracaibo. Abhandlung der Naturforschungs Geschichte in Halle, Sitzungsberichte 2: 15-17.

Cabrera, A. 1961. Catálogo de los mamíferos de América del Sur. Comun. Mus. Argent. Cienc. Nat. „Bernardino Rivadavia“ Inst. Nac. Invest. Cienc. Nat. (Argent.) Zool. 4: 309-732.

Cameron, G.N. \& S.R. Spencer. 1981. Sigmodon hispidus. Mammal. Species 158: 1-9.

Carpenter, J. 1996. Uninformative Boostraping. Cladistics 12: $177-181$.

Carroll, D.S. \& R. Bradley. 2005. Systematics of the genus Sigmodon: DNA sequences from beta-fibrinogen and cytochrome b. Southwest. Nat. 50: 342-349.

Carroll, D.S., L.L. Peppers \& R.D. Bradley. 2005. Molecular systematics and phylogeography of the Sigmodon hispidus species group, p. 87-100. In V. Sánchez-Cordedor \& R.A. Medellín (eds.). Instituto de Biología e 
Instituto de Ecología, Universidad Nacional Autónoma de México y Comisión Nacional el Conocimiento y Uso de la Biodiversidad, Conario, México.

D’Elía, G. 2003. Phylogenetics of Sigmodontinae (Rodentia. Muroidea. Cricetidae) with special reference to the akodont group and with additional comments on historical biogeography. Cladistics 19: 307-323.

Dalby, P.L. \& H.A. Lillevik. 1969. Taxonomic analysis of electrophoretic blood serum patterns in the cotton rat. Sigmodon. Publ. Mus., Michigan State Uni., Biol. Ser. 4: 65-101.

De Lima, H., Z. De Guglielmo, A. Rodríguez, J. Convit \& N. Rodríguez. 2002. Cotton rats (Sigmodon hispidus) and black rats (Rattus rattus) as possible reservoirs of Leishmania spp. in Lara state. Venezuela. Mem. Inst. Oswaldo Cruz 97: 169-174.

De Lima, H., J. Carrero, A. Rodríguez, Z. De Guglielno \& N. Rodríguez. 2006. Trypanosomatidae de importancia en salud pública en animales silvestres y sinantrópicos en un área rural del municipio Tovar del estado Mérida, Venezuela. Biomédica 26: 42-50.

Dickerman, A.W. 1992. Molecular systematics of some new world murid rodents. Ph.D. Thesis. University of Wisconsin, Madison, EEUU.

Geise, L., M.F. Smith \& J.L. Patton. 2001. Diversification in the genus Akodon (Rodentia: Sigmodontinae) in Southeastern South America: mitochondrial DNA sequence anlysis. J. Mammal. 82: 92-101.

Golczer, G. \& J. Arrivillaga. 2008. Modificación de un protocolo estándar de extracción de ADN para flebotominos pequeños (Phlebotominae: Lutzomyia). Rev. Colomb. Entomol. 34: 199-202.

Golczer, G. \& J. Arrivillaga. 2010. Gen period no construye filogenias dentro del complejo de species, Lutzomyia longipalpis (Diptera: Phlebotominae). MES. 2: $10-26$.

Hoffmann, F.G., E.P. Lessa \& M.F. Smith. 2002. Systematics of Oxymycterus with description of a new species from Uruguay. J. Mammal. 83: 408-420.

Irwin, D.M., T.D. Kocher \& A.C. Wilson. 1991. Evolution of cytochrome $b$ gene in mammals. J. Mol. Evol. 2: 37-55.

Kocher, T.D., W.K. Thomas, A. Meyer, S.V. Edwards, S. Paabo, F.X. Villablancatt \& A.C. Wilson. 1989. Dynamics of mitochondrial DNA evolution in animals: amplification and sequencing with conserved primers (cytochrome b/12S ribosomal DNA/control region/evolutionary genetics/molecular phylogenies). Proc. Natl. Acad. Sci. Unit. States. Am. 86: 6196-6200.

Lavrenchenko, L.A. \& E. Verheyen. 2006. Evolutionary relationships among Narrow-Headed rats (genus Stenocephalemys, Muridae, Rodentia) inferred from complete Cytochrome $b$ gene sequences. Russ. J. Genet. 42: 439-446.

Leite, Y.L.R. \& J.L. Patton. 2002. Evolution of South American spiny rats (Rodentia. Echimyidae): the star-phylogeny hypothesis revisited. Mol. Phylogenet. Evol. 25: 455-464.

Linares, O.J. 1998. Mamíferos de Venezuela. Sociedad Conservacionista Audubon de Venezuela, Caracas, Venezuela.

Musser, G.G., M.D. Carlenton. 2005. Srperfamilia Muroidea, p. 894-1535. In D. Wilson \& D.M. Reeder (eds). Mammal species of the world. A taxonomic and geographic reference. Johns Hopkins University, Baltimore, EEUU.

Myers, P., B. Lundrigan \& P.K. Tucker. 1995. Molecular phylogenetics of oryzomyine rodents: the genus Oligoryzomys. Mol. Phylogenet. Evol. 4: 372-382.

Patton, J.L. \& M.F. Smith. 1992. mtDNA Phylogeny of andean mice: A test of diversification across ecological gradients. Evolution 46: 174-183.

Peppers, L.L. \& R. Bradley. 2000. Cryptic species in Sigmodon hispidus: Evidence from DNA sequences. J. Mammal. 81: 332-343.

Peppers, L.L., D.S. Carroll \& R. Bradley. 2002. Molecular systematics of the genus Sigmodon (Rodentia: Muridae): Evidence from the mitochondrial cytochrome-b gene. J. Mammal. 83: 396-407.

Say, T. \& G. Ord. 1825. Description of a new species of Mammalia. whereon a new genus is proposed to be found. J. Acad. Nat. Sci. Phila. 4: 352-355.

Smith, M. \& J. Patton. 1999. Phylogenetic relationships and the radiation of Sigmodontine rodents in South America: evidence from Cytochrome $b$. J. Mammal. Evol. 6: 89-125.

Sullivan, J., J.A. Market \& C.W. Kilpatrick. 1997. Phylogeography and molecular systematics of the Peromyscus aztecus species group (Rodentia: Muridae) inferred using parsimony and likelihood. Syst. Biol. 46: 426-440. 
Swofford, D.L. 2001. PAUP: Phylogenetic Analysis Using Parsimony (and others methods) version 4. Sinauer, Sunderland, Massachusetts, EEUU.

Tajima, F. 1993. Simple methods for testing molecular clock hypothesis. Genetics 135: 599-607.

Tamura, K. \& M. Nei. 1993. Model selection in the estimation of the number of nucleotide susbtitutions. Mol. Biol. Evol. 10: 512-526.

Tamura K., J. Dudley, M. Nei \& S. Kumar. 2007. MEGA4: Molecular Evolutionary Genetics Analysis (MEGA) software version 4.0. Mol. Biol. Evol. 24: 1596-1599.
Thomas, O. 1914. Four new mammals species from Venezuela. Ann. Mag. Nat. Hist. 8: 410-414.

Thompson, J.D., D.G. Higgins \& T.J. Gibson. 1994. CLUSTAL W: improving the sensitivity of progressive multiple sequence alignment through sequence weighting, positions-specific gap penalties and weight matrix choice. Nucleic Acids Res. 22: 4673-4680.

Voss, R.S. 1992. A revision of the South American species of Sigmodon (Mammalia: Muridae) with notes on their natural history and biogeography. Am. Mus. Novit. 3050: 1-56. 